Research Paper

\title{
Bisphenol A, Dichlorodiphenyltrichloroethane (DDT) and Vinclozolin Affect ex-vivo Uterine Contraction in Rats via Uterotonin (Prostaglandin F2 $\alpha$, Acetylcholine and Oxytocin) Related Pathways
}

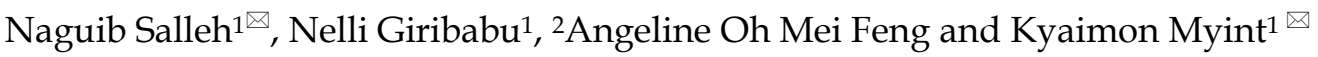 \\ 1. Department of Physiology, Faculty of Medicine, University of Malaya, Lembah Pantai, 50603 Kuala Lumpur, Malaysia; \\ 2. Department of Biomedical Science, Faculty of Health and Life Sciences, Management and Science University, Shah Alam, 40100 Selangor Darul \\ Ehsan, Malaysia. \\ $\square$ Corresponding authors: naguib.salleh@yahoo.com.my, kyaimon@um.edu.my, Tel: 0060172082715 Fax: 00603-7967 7532
}

(C) 2015 Ivyspring International Publisher. Reproduction is permitted for personal, noncommercial use, provided that the article is in whole, unmodified, and properly cited. See http://ivyspring.com/terms for terms and conditions.

Received: 2015.02.22; Accepted: 2015.09.02; Published: 2015.10.27

\begin{abstract}
Bisphenol-A (BPA), dichrolodiphenyltrichloroethane (DDT) and vinclozolin were found able to induce abnormal uterine contraction. The mechanisms involved remains unclear. We hypothesized that the effect of these compounds were mediated via the uterotonin pathways. Therefore, in this study, effects of BPA, vinclozolin and DDT-only and in combination with uterotonins (PGF-2 $\alpha$, acetylcholine and oxytocin) on the force and pattern of uterine contraction were observed. Methods: Uteri were harvested from intact adult female rats 24 hours after a single injection $(1 \mathrm{mg} / \mathrm{kg} / \mathrm{b} . \mathrm{w})$ of estrogen to synchronize their oestrous cycle. The uterine horns were subjected for ex-vivo contraction studies in an organ bath connected to Powerlab data acquisition system. Different doses of BPA, vinclozolin and DDT were added into the bathing solution and changes in the pattern and strength of uterine contraction were recorded. Further, increasing doses of uterotonins were concomitantly administered with these compounds and changes in the force and pattern of contraction were observed. Results: In the absence of uterotonins, uterine contractile force decreased with increasing doses of BPA and DDT. However, vinclozolin induced sharp increase in the contractile forces which then gradually decrease. Administration of BPA, DDT and vinclozolin alone reduced the force of uterine contraction following stimulation of contraction by uterotonins. However, BPA, vinclozolin or DDT effects were relieved upon co-administration with uterotonins at increasing doses. Conclusions: The antagonizing effect of uterotonins on BPA, vinclozolin and DDT actions could explain the mechanism underlying the adverse effect of these compounds on uterine contraction.
\end{abstract}

Key words: bisphenol A, vinclozolin, DDT, uterine contractility, uterotonins.

\section{Introduction}

Contraction of the uterus is important to facilitate multiple reproductive processes including sperm and ovum transport and fetal and placental expulsion [1]. Uterine contractility was low throughout pregnancy which confer protection against abortion and premature delivery [2]. Contractility of uterus changes throughout the reproductive cycle. High frequency contraction was observed near ovulation while low frequency contraction was observed following ovulation [3]. Contractility of uterine smooth muscle can be controlled by hormonal and neural factors examples progesterone, oxytocin and prostaglandin $\mathrm{F} 2 \mathrm{a}$ (PGF2a) (hormonal) and acetylcholine (neural). These factors bind to their respective receptors on the uterine smooth muscle cell membrane which then trigger series of intracellular events leading to increase in 
intracellular $\mathrm{Ca}^{2+}$ levels that initiates contraction [4]. Several compounds have been reported able to affect uterine contraction, thus could interfere with the normal uterine reproductive processes. BPA, vinclozolin and DDT are the common endocrine disrupting chemicals (EDCs) which are found able to induce abnormal uterine contraction.

Bisphenol-A (BPA) is an organic compound widely used in the making of polycarbonate polymers, epoxy resins and plastics [5]. BPA was found able to induce changes in the expression of key developmental genes in the pregnant mouse uteri [6]. In rats, exposure to high dose BPA $(100 \mathrm{mg} / \mathrm{kg} /$ day $)$ could affect establishment of uterine receptivity [7]. This could result in reduced embryo implantation rate [8]. In humans, a positive dose-response relationship was observed between urinary BPA concentration and failure of the embryo to implant [9]. There were evidences that BPA could stimulate uterine contraction via oxytocin-related pathway in immature rats [10]. Vinclozolin, a fungicide widely applied on fruits, vegetables, and ornamental plants [11], has been reported to interfere with multiple uterine functions. Female rats exposed to vinclozolin were found to have excessive uterine weight and levels of serum luteinizing hormone (LH) and androstenedione [12]. In addition, in these animals, high level of ovarian androstenedione and estrone were also observed [12]. However, this compound did not disrupt ovulation and expression of progesterone receptors in the uterus of cycling female rats [13]. Vinclozolin has been reported to reduce the pregnancy duration, increase the abortion percentage, decrease the number of pregnancies and decrease the number of juveniles per females in lice (Porcellio scaber) [14]. In view of these, there were possibilities that vinclozolin could interefere with uterine contraction.

Dichrolodiphenyltrichloroethane (DDT), an organochlorine pesticide, could be found in almost every conceivable forms including solution, powder, vaporizer and lotion [15]. Exposure to DDT has been linked to early pregnancy loss. A prospective study in humans has revealed a positive association between pre-conception serum DDT level and risk of early pregnancy loss [16]. Studies have found that DDT effects could be passed through generations i.e. from mother to offspring. Mothers exposed to high concentration of DDT have high number of offspring with difficulty to conceived [17]. The detail mechanisms underlying DDT effect on the uterus however remain largely unknown.

In view that BPA, vinclozolin and DDT were reported able to induce abnormal contraction of the uterus, we hypothesized that these compound could interfere the normal physiological mechanisms that regulate uterine contraction. We further hypothesized that these compounds' effect involved the uterotonin (PGF2a, acetylcholine and oxytocin) pathways. The aims of this study were to investigate the involvement of uterotonin pathways in mediating BPA, vinclozolin and DDT effects on uterine contraction which could help to elucidate the mechanisms underlying these compounds' adverse effects on multiple reproductive processes in uterus.

\section{Materials and Methods}

\section{Uterine tissue preparation}

Adult female Sprague-Dawley (SD) rats weighing 220-250 g were purchased from animal unit, University of Malaya. The rats were housed in a controlled environment with temperature $25 \pm 2{ }^{\circ} \mathrm{C}$, relative humidity of between $30-70 \%, 12$ hours light-dark cycle and had free access to rodent food pellet and tap water ad libitum. All chemicals used were of analytical grades. Intact female rats were treated with high dose of $17 \beta$ estradiol benzoate (Sigma-Aldrich, USA) at $1 \mathrm{mg} / \mathrm{kg} / \mathrm{b} . \mathrm{w}$ to synchronize their oestrous cycle [18]. A day after injection, rats were sacrificed and uteri were immediately removed and placed in a physiological solution. All experimental procedures were approved by Institutional Animal Care and Use Committee, University of Malaya.

\section{Ex-vivo uterine contraction study}

The harvested uterine horns were cut open vertically and placed into an organ bath pre-filled with physiological solution with the following electrolytes composition: $\mathrm{NaCl}(155 \mathrm{mM}), \mathrm{KCl}(4.5 \mathrm{mM}), \mathrm{MgCl}_{2}$ (1.0 mM), $\mathrm{CaCl}_{2}(2.0 \mathrm{mM})$ and D- glucose $(10 \mathrm{mM}) \cdot \mathrm{pH}$ of the solution was adjusted to 7.40 by adding $\mathrm{NaOH}$. Temperature of the organ bath was maintained at $37^{\circ} \mathrm{C}$. $95 \% \mathrm{O}_{2}$ and $5 \% \mathrm{CO}_{2}$ was continuously delivered into the bathing solution. Each strip of uterine horns was placed under optimum resting force of $1 \mathrm{~g}$ and was allowed to equilibrate for 30 minutes prior to drug administration. During this period, uterine strips were washed with $10 \mathrm{ml}$ of fresh physiological solution every 15 minutes according to the method as described by Oropeza et al., [19]. Each experiment was repeated six times by using new uterine strips from different rats $(n=6)$. Contractile forces were recorded isometrically by using a force transducer which was connected to a bridge amplifier and to the PowerLab data acquisition system (ADI Instrument, Australia).

Bisphenol-A, vinclozolin and DDT (Sigma Aldrich Co, MO, USA) were added into the organ bath in a non-cumulative, dose-dependent manner $\left(1 \times 10^{-8}\right.$ to $1 \times 10^{-4} \mathrm{M}$ ). In between administration of different drugs and different doses of the same drug, the 
bathing solution was flushed-out from the organ bath and immediately replaced with a new solution containing the desired drug or desired dose of the same drug. Subsequently, the response of the uterus were recorded. The exposure time of each of the uterine strip to the drug was between 3 - 5 minutes. Preliminary investigation revealed that $1 \times 10^{-2} \mathrm{M}$ Ach (Sigma-Aldrich, USA), $1 \times 10^{-6} \mathrm{M}$ oxytocin (Sigma-Aldrich, USA) and $1 \times 10^{-6}$ M PGF2a (Tocris Bioscience, USA) produced the maximum force of uterine contraction. In order to investigate the involvement of uterotonin-related pathways in mediating BPA, vinclozolin and DDT effects on uterine contraction, these compounds were introduced in the organ bath with uterine strip pre-exposed to uterotonins (PGF2a, acetylcholine and oxytocin). Later, uterotonins were added at increasing doses and ability of uterotonins to antagonize BPA, vinclozolin and DDT effects were then recorded. The drugs were added into the bathing solution with no washing was performed in-between the different tests.

\section{Statistical analysis}

Results were expressed as mean \pm SEM from 6 uterine strips of 6 different animals. Statistical significance was evaluated by one-way analysis of variance (ANOVA) by using SPSS version 7.5 (SPSS, Cary, NC, USA) and the individual comparisons were obtained by Duncan's multiple range test (DMRT). $P<0.05$ was considered as statistically significant.

\section{Results}

\section{Effects of BPA, vinclozolin and DDT on uterine contraction ex-vivo}

Figure 1A shows dose-dependent effect of BPA on uterine contraction. The average maximum contractile force prior to exposure to BPA was $0.17 \pm 0.08 \mathrm{~g}$ tension. Following exposure to $10^{-8} \mathrm{M}$ BPA, the mean maximum uterine contractile force was significantly reduced. Exposure to $10^{-6} \mathrm{M}$ BPA further decreased the uterine contractile forces. Following exposure of $10^{-4} \mathrm{M} \mathrm{BPA}$, maximum force of contraction was approximately two times lower compared to the maximum force generated in uterus non-exposed to BPA.

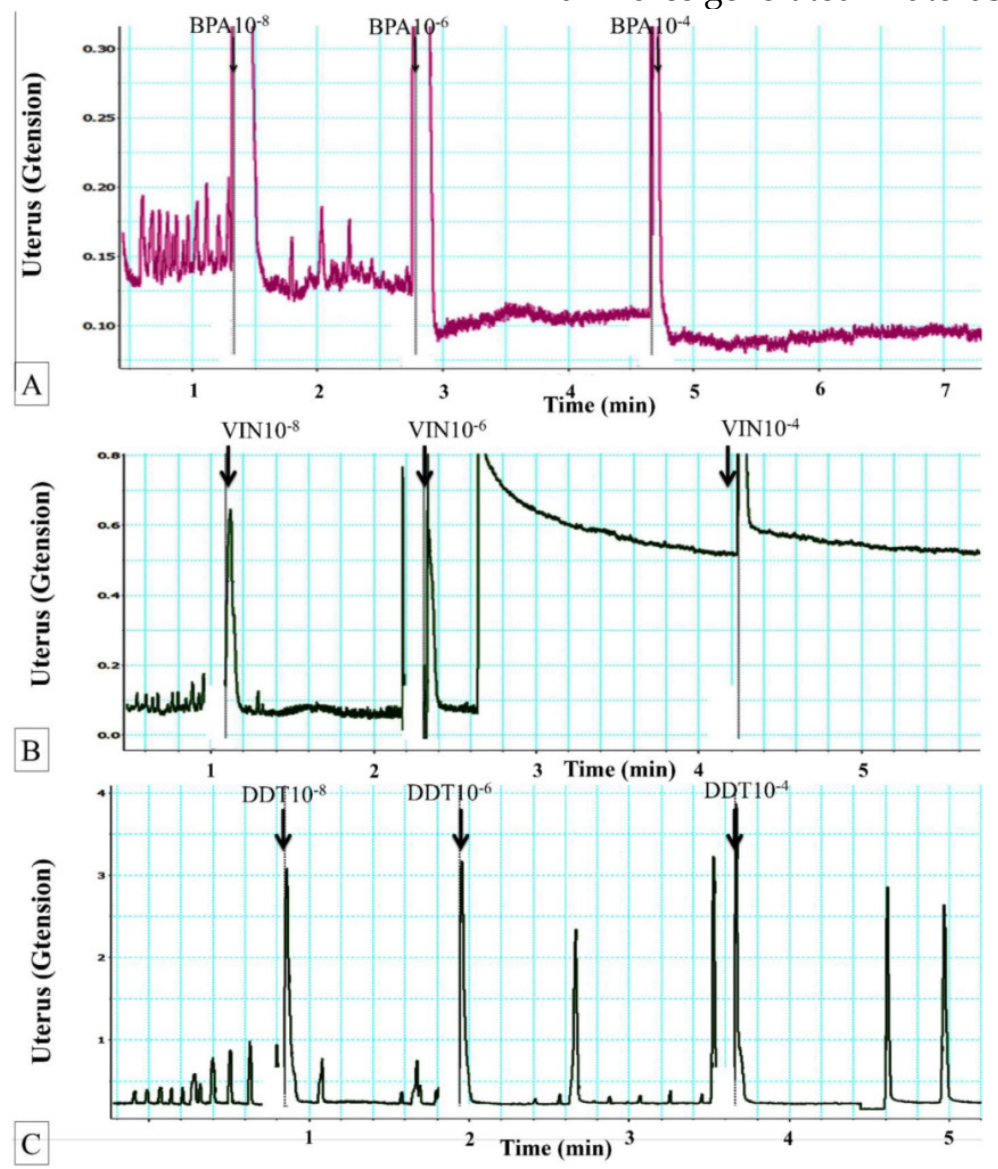

Figure 1. (A) Dose-dependent effect of BPA on uterine contraction. Representative tracing of dose-dependent effect of BPA on uterine contraction. Exposure to 10-6 $M$ BPA resulted in marked decrease in the contractile forces. Regular contraction was noted following BPA exposure. BPA: bisphenol A. (B) Dose-dependent effect of vinclozolin on uterine contraction. Representative tracing of vinclozolin effect on uterine contraction. Exposure to 10-6 M vinclozolin resulted in elevated baseline contractile force followed by progressive decrease in the force of contraction. Regular pattern of contraction was observed following vinclozolin exposure. (C) Dose-dependent effect of DDT on uterine contraction. Representative tracing of DDT effect on uterine contraction. Exposure to DDT at doses above $10^{-6} \mathrm{M}$ resulted in a decrease in uterine contractile force. Meanwhile, regular pattern of contraction was observed following DDT exposure. 


\section{Effects of vinclozolin on uterine contraction}

In figure $1 \mathrm{~B}$, a sudden, markedly elevated baseline contractile force was observed following $10^{-6} \mathrm{M}$ vinclozolin exposure. Following this, the force steadily decreased. Similar decrease in the contractile force was also observed following exposure to $10^{-4} \mathrm{M}$ vinclozolin. Following vinclozolin exposure, the pattern of contraction was mostly regular.

\section{Effects of DDT on uterine contraction}

In figure $1(C)$, exposure to increasing doses of DDT resulted in decreased in the force of contraction. A few low magnitude contractile responses were observed in between the peaks. Following exposure to $10^{-4} \mathrm{M}$ DDT, the contractile forces generated was nearly zero. Mixed regular and irregular contraction patterns were noted following DDT exposure.

\section{Mechanisms underlying BPA effect on uterine contraction}

Involvement of PGF2 $\alpha$ pathway

In figures $2(\mathrm{~A}) \&(\mathrm{~B})$, the force of uterine contraction induced by $10^{-6} \mathrm{M}$ PGF2 $\alpha$ was significantly reduced following administration of $10^{-6} \mathrm{M}$ BPA (four-folds lower compared to PGF2a alone). Following exposure to $10^{-10} \mathrm{M}$ PGF2 $\alpha$, the force of uterine contraction was higher when compared to BPA alone. Administration of $10^{-8} \mathrm{M}$ PGF2 $\alpha$ resulted in approximately two and a half folds greater force of contraction when compared to BPA alone. Exposure to higher doses of PGF2 $\alpha(>10-6 \mathrm{M})$ with BPA did not result in further increase in the uterine contraction forces when compared to combined $10^{-8} \mathrm{M}$ PGF2 $\alpha$ and BPA exposure. Throughout the experiment, regular pattern of contraction was observed.

Involvement of Ach pathway

In figures $3(\mathrm{~A})$ \& $(\mathrm{B})$, the force of uterine contraction was highest following exposure to $1 \times 10^{-2} \mathrm{M}$ Ach alone. Following exposure to BPA, uterine contractile forces were markedly reduced. In uteri exposed to $10^{-6} \mathrm{M}$ BPA, a dose-dependent increase in the contractile force was observed with increasing dose of acetylcholine (from $10^{-10}$ to $10^{-2} \mathrm{M}$ ). The contractile force was markedly increased following exposure to $10^{-4} \mathrm{M}$ Ach and BPA. Throughout the experiment, regular pattern of uterine contraction was observed.

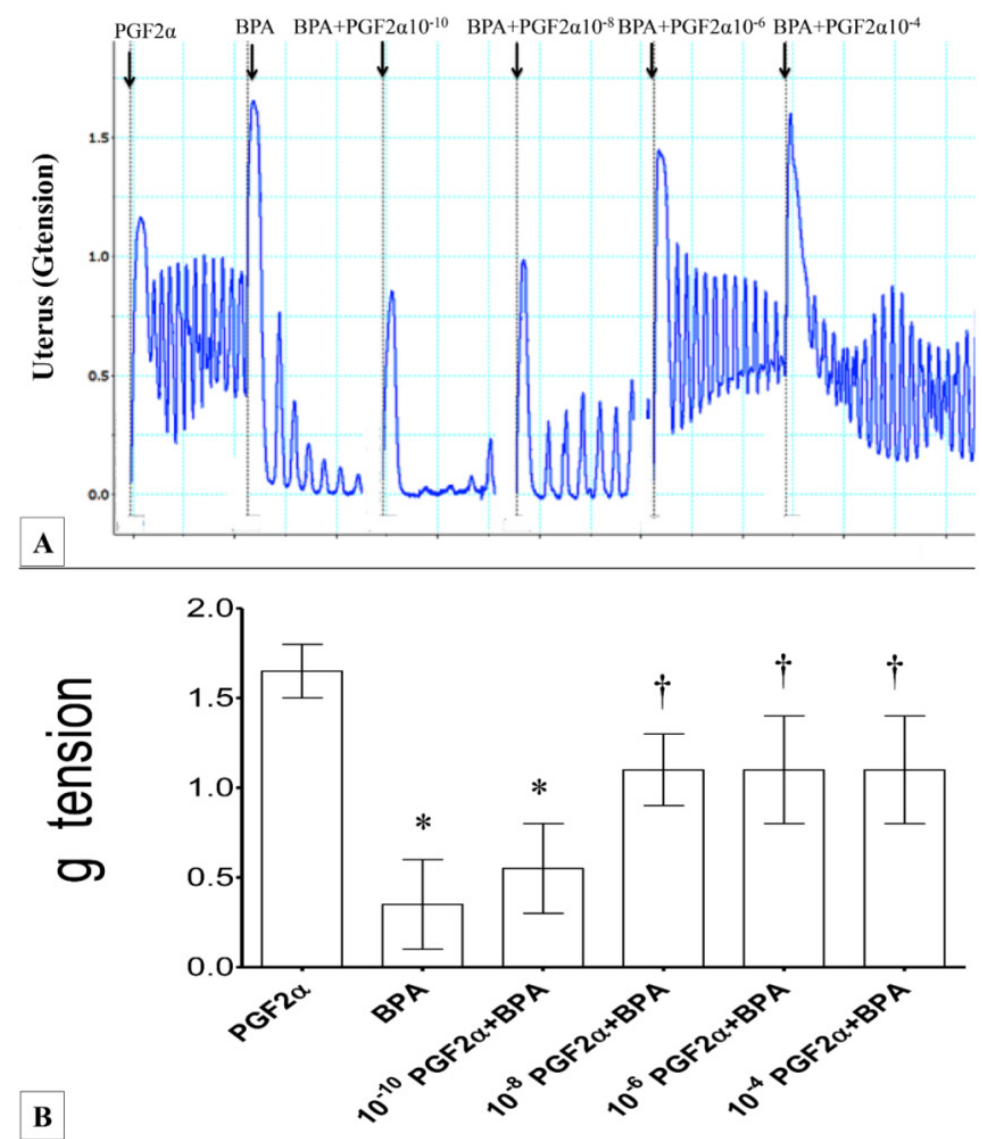

Figure 2. (A) \& (B): Combined effect of PGF2 $\alpha$ and BPA on uterine contraction. Exposure to 10-6M PGF2 $\alpha$ alone resulted in highest force of contraction. Subsequently, addition of BPA resulted in markedly reduced uterine contractile forces. The effect of BPA was antagonized following co-administration of increasing doses of PGF2 $\alpha$ $\left(10^{-8}\right.$ to $\left.10^{-4} \mathrm{M}\right) * p<0.05$ compared to PGF2 $\alpha$ alone. $\dagger p<0.05$ compared to bisphenol $A$ alone. $n=6$ animals per group. BPA: bisphenol A, P: PGF2 $\alpha$. 

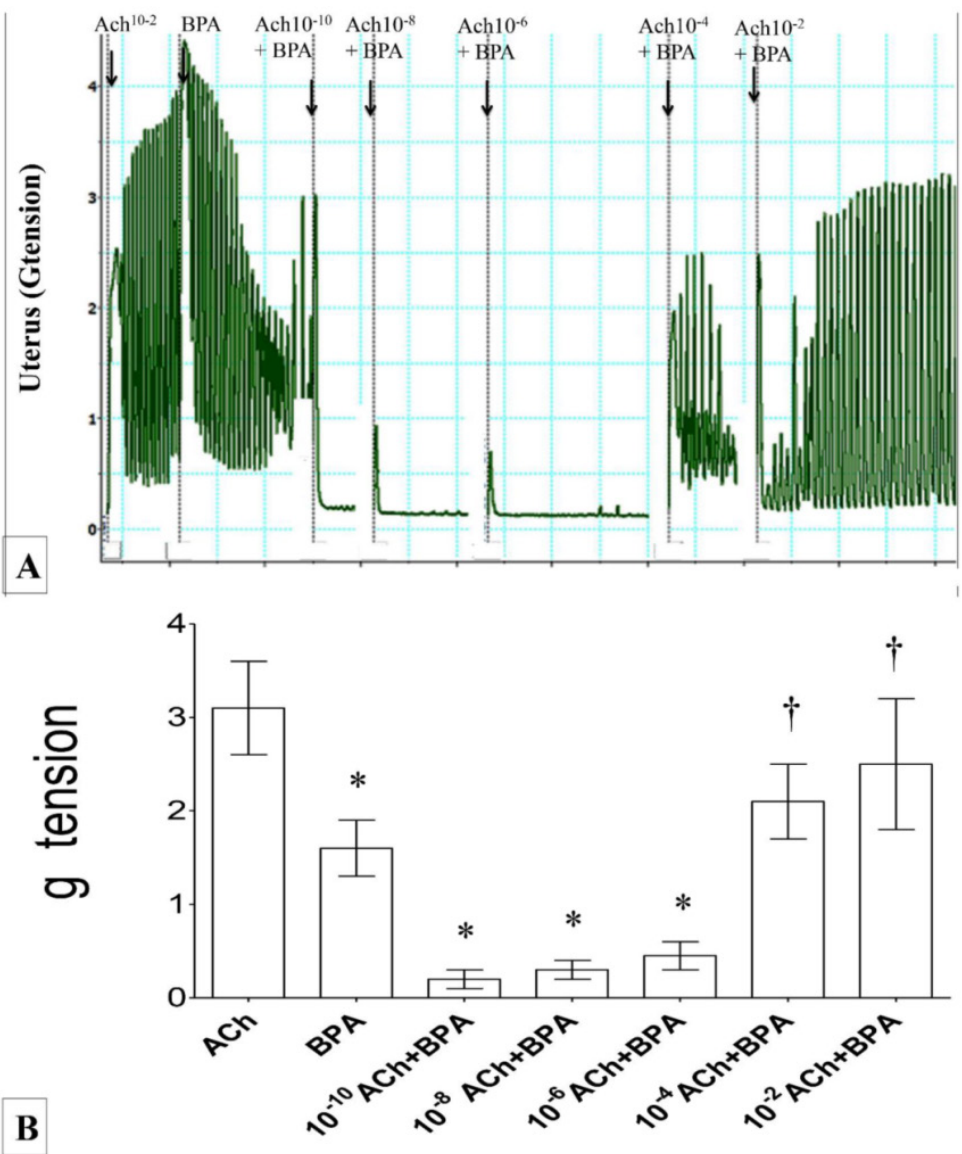

Figure 3. (A) \& (B): Combined effect of Ach and BPA on uterine contraction. High contractile force was observed following exposure to 10-2 M Ach. Administration of 10-6M BPA significantly reduced the contractile force. A dose-dependent increase in uterine contractile force was observed following combined administration of BPA with increasing doses of Ach. ${ }^{*} p<0.05$ as compared to ACh alone. $\dagger p<0.05$ as compared to bisphenol $A$ alone. $n=6$ animals per group ACh: acetylcholine, BPA: bisphenol A.

\section{Involvement of oxytocin pathway}

In figures $4(\mathrm{~A})$ \& (B), high force of contraction was observed following exposure to $10^{-6} \mathrm{M}$ oxytocin. Administration of BPA alone reduced the force of uterine contraction by approximately one and half time compared to $10^{-6} \mathrm{M}$ oxytocin. Exposure to increasing dose of oxytocin (from $10^{-10}$ to $10^{-6} \mathrm{M}$ ) in the presence of BPA resulted in progressive increase in uterine contractile force. Irregular pattern of contraction was observed in uterus throughout the experiment.

\section{Mechanisms underlying vinclozolin effect on uterine contraction}

\section{Involvement of PGF2 $\alpha$ pathway}

In figures 5(A) \& (B), exposure 10-6 M PGF2a resulted in highest force of uterine contraction. Exposure to vinclozolin at $10^{-6} \mathrm{M}$ resulted in a significant decrease in the force of contraction. Concomitant administration of vinclozolin with $10^{-10}$ to $10^{-8} \mathrm{M}$ PGF2 $\alpha$ resulted in a dose-dependent increase in the uterine contractile forces. Concomitant exposure to vinclozolin and $10^{-6} \mathrm{M}$ PGF2a resulted in two fold higher contractile forces when compared to vinclozolin alone. Beyond this dose, no further increase in contractile forces was observed. Regular pattern of contraction was observed throughout the experiment.

\section{Involvement of Ach pathway}

In figures $6(\mathrm{~A}) \& \mathrm{~B}$, the highest force of uterine contraction was observed following administration of 10-2 M Ach. The contractile forces were markedly reduced following administration of vinclozolin. However, co-administration of increasing dose of Ach $\left(10^{-10}\right.$ to $\left.10^{-6} \mathrm{M}\right)$ and vinclozolin resulted in a dose-dependent increase in the force of uterine contraction. Administration of Ach beyond $10^{-4} \mathrm{M}$ resulted in maximum force of uterine contraction. Regular pattern of contraction was observed throughout the experiment. 


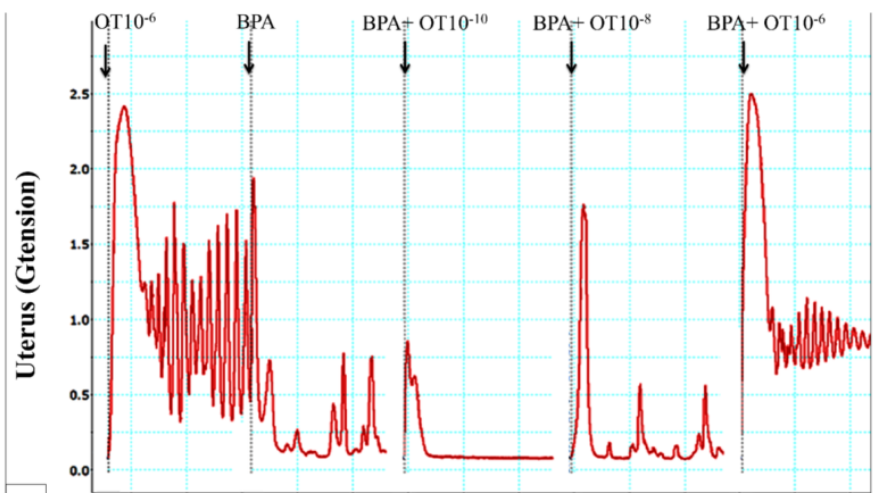

A

B

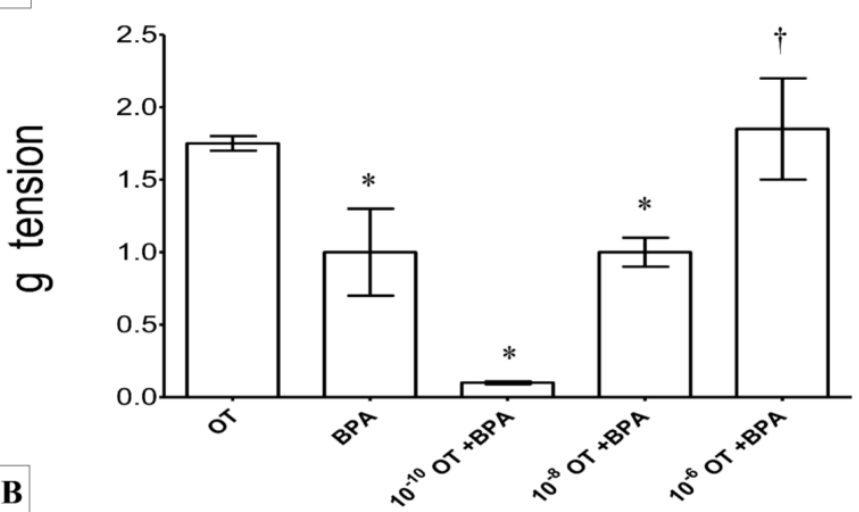

Figure 4. (A) \& (B): Combined effect of oxytocin and BPA on uterine contraction. High force of contraction was observed following exposure to 10-6M oxytocin. The force was reduced following administration of BPA. Dose-dependent increase in uterine contractile force was observed following co-exposure of BPA with increasing doses of oxytocin (10-10 to 10-6M). * $p<0.05$ as compared to oxytocin alone. $\dagger p<0.05$ as compared to BPA alone. $n=6$ animals per group BPA: bisphenol A, OT: oxytocin.

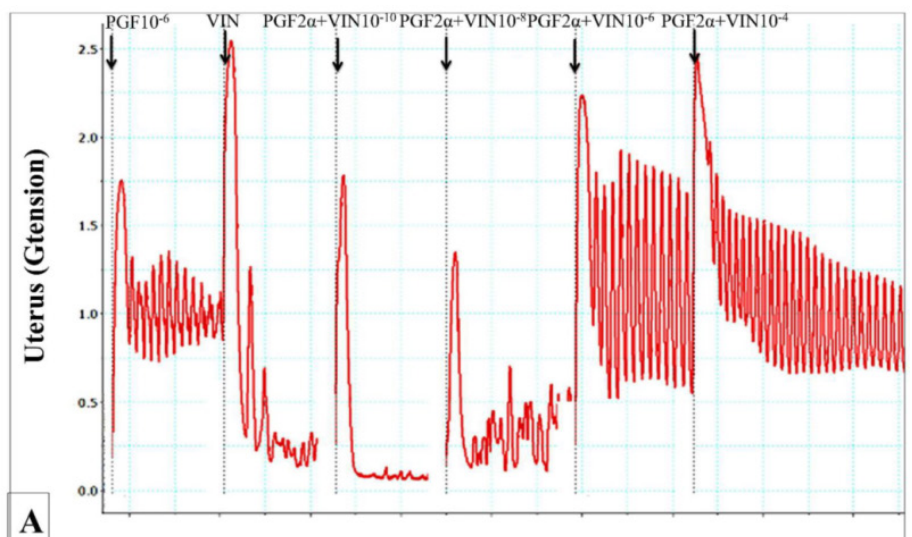

$\mathbf{A}$

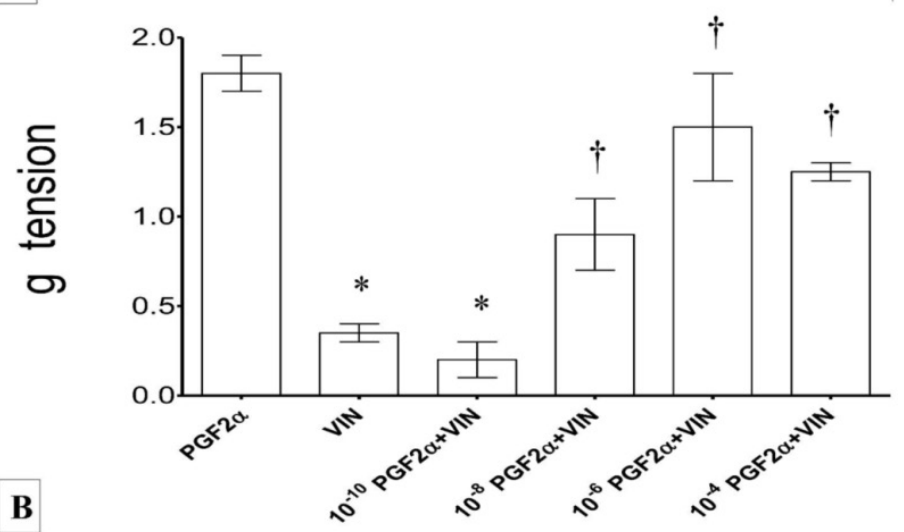

Figure 5. (A) \& (B): Combined effect of PGF2 $\alpha$ and vinclozolin on uterine contraction. Exposure to 10-6M PGF2 $\alpha$ alone caused highest force of contraction. Exposure to vinclozolin resulted in reduced in uterine contractile force which was progressively increased with increasing dose of PGF2 $\alpha * p<0.05$ as compared to PGF2 $\alpha$ alone. $\dagger p<0.05$ as compared to vinclozolin alone. $n=6$ animals per group P: PGF2 $\alpha$, V/VIN: vinclozolin. 

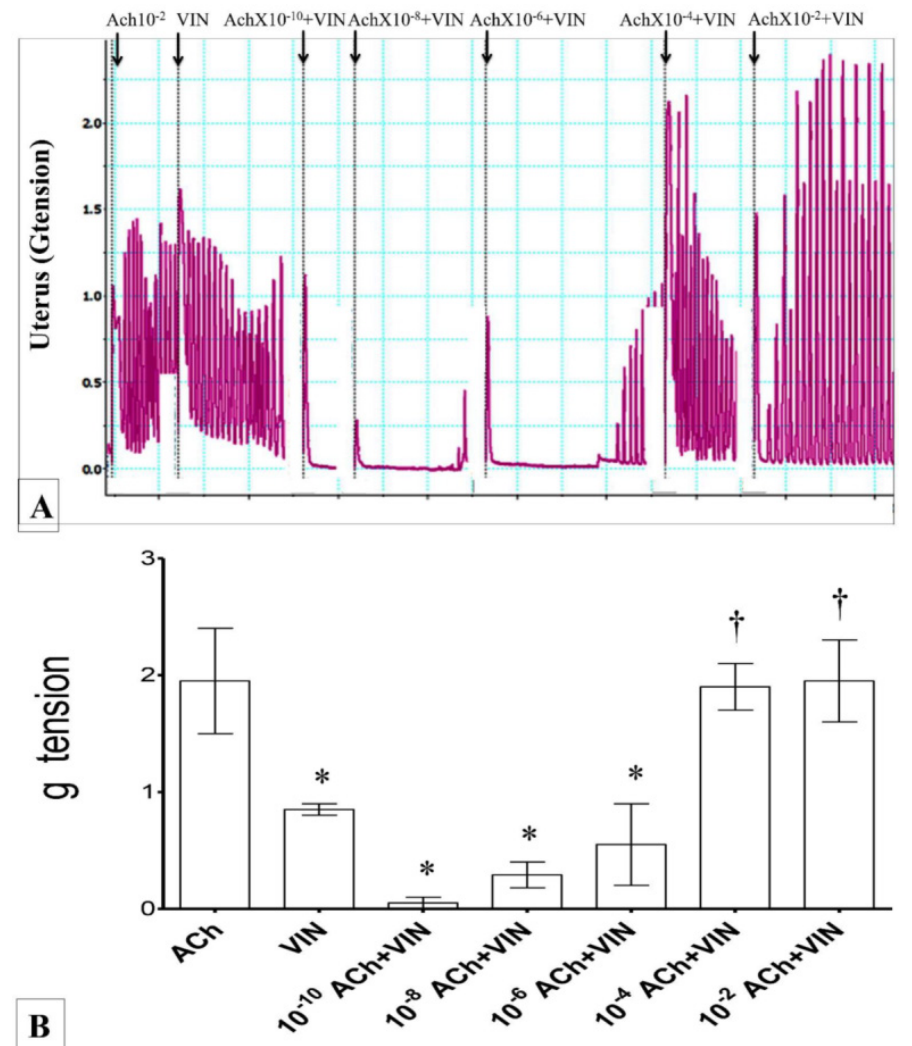

Figure 6. (A) \& (B): Combined effect of Ach and vinclozolin on uterine contraction. Highest force of contraction was noted following exposure to 10-2M Ach. Exposure to vinclozolin resulted in marked decrease in the uterine contractile force. Force of uterine contraction increased with increasing doses of Ach. $* p<0.05$ as compared to Ach. $\dagger p<0.05$ compared to vinclozolin $n=6$ animals per group Ach: acetylcholine, Vin: vinclozolin.

\section{Involvement of oxytocin pathway}

In figures $7(\mathrm{~A}) \&(\mathrm{~B})$, administration of $10^{-6} \mathrm{M}$ oxytocin alone resulted in highest force of contraction. Administration of vinclozolin caused a significant decrease in the uterine contractile forces. Combined administration of vinclozolin with increasing doses of oxytocin resulted in a progressive increase in uterine contractile forces. Exposure to vinclozolin with 10-6 M oxytocin resulted in a remarkable increase in uterine contractile force (four-folds higher than viclozolin alone). This was not significantly different when compared to $10^{-6} \mathrm{M}$ oxytocin alone. Throughout the experimental period, mixed regular and irregular patterns of contraction were observed.

\section{Mechanisms underlying DDT effect on uterine contraction}

\section{Involvement of PGF2 $\alpha$ related pathway}

In figures $8(\mathrm{~A})$ \& $(\mathrm{B})$, the force of uterine contraction was highest following exposure to $10^{-6} \mathrm{M}$ PGF2a. Uterine contractile force decreased significantly following exposure to DDT. In DDT-exposed uteri, co-administration of PGF2a at an increasing doses resulted in increased force of contraction. The highest force was achieved following combined exposure to $10^{-6} \mathrm{M}$ PGF2a with DDT. The pattern of contraction observed throughout the experiment was mostly regular.

\section{Involvement of Ach-related pathway}

In figures $9(\mathrm{~A})$ \& (B), exposure to $10^{-2} \mathrm{M}$ Ach resulted in highest uterine contractile force. Exposure to DDT caused a marked reduction in the force of uterine contraction. In rats exposed to DDT with increasing doses of Ach, the force of contraction was markedly increased. A maximum force of contraction was achieved following exposure to 10-4M Ach and DDT. The pattern of uterine contraction following DDT exposure was mostly regular.

\section{Involvement of oxytocin-related pathways}

In figures $10(\mathrm{~A}) \&(\mathrm{~B})$, the highest force of uterine contraction was observed following exposure to $10^{-6} \mathrm{M}$ oxytocin. Markedly reduced uterine contractile forces were observed following exposure to DDT. Co-administration of DDT with oxytocin in a dose-dependent manner resulted in a progressive increase in the force of uterine contraction. Exposure to $10^{-6} \mathrm{M}$ oxytocin and DDT resulted in the highest force of uterine contraction which was not significantly different compared to exposure to $10^{-6} \mathrm{M}$ oxytocin alone. Throughout the experiment, mixed regular and irregular contraction patterns were observed. 


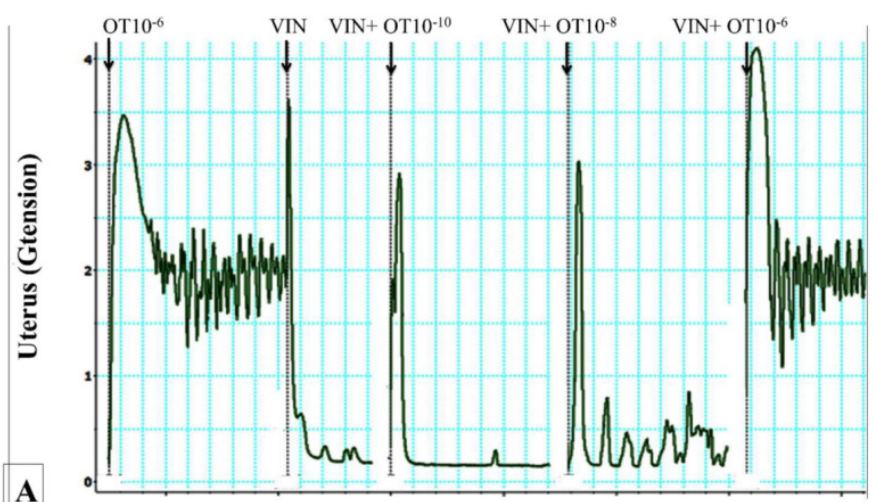

A

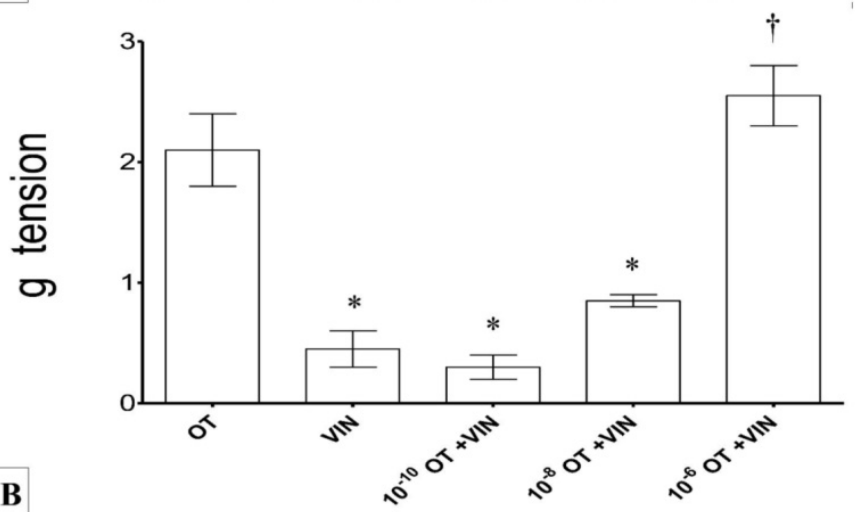

Figure 7. (A) \& (B): Combined effects of oxytocin and vinclozolin on uterine contraction. Exposure to 10-6 $\mathrm{M}$ oxytocin resulted in highest force of contraction. An increase in the force was observed with increasing dose of oxytocin and vinclozolin. $* p<0.05$ as compared to Ach. $\dagger p<0.05$ compared to oxytocin. $n=6$ animals per group OT: oxytocin, VIN: vincozolin.
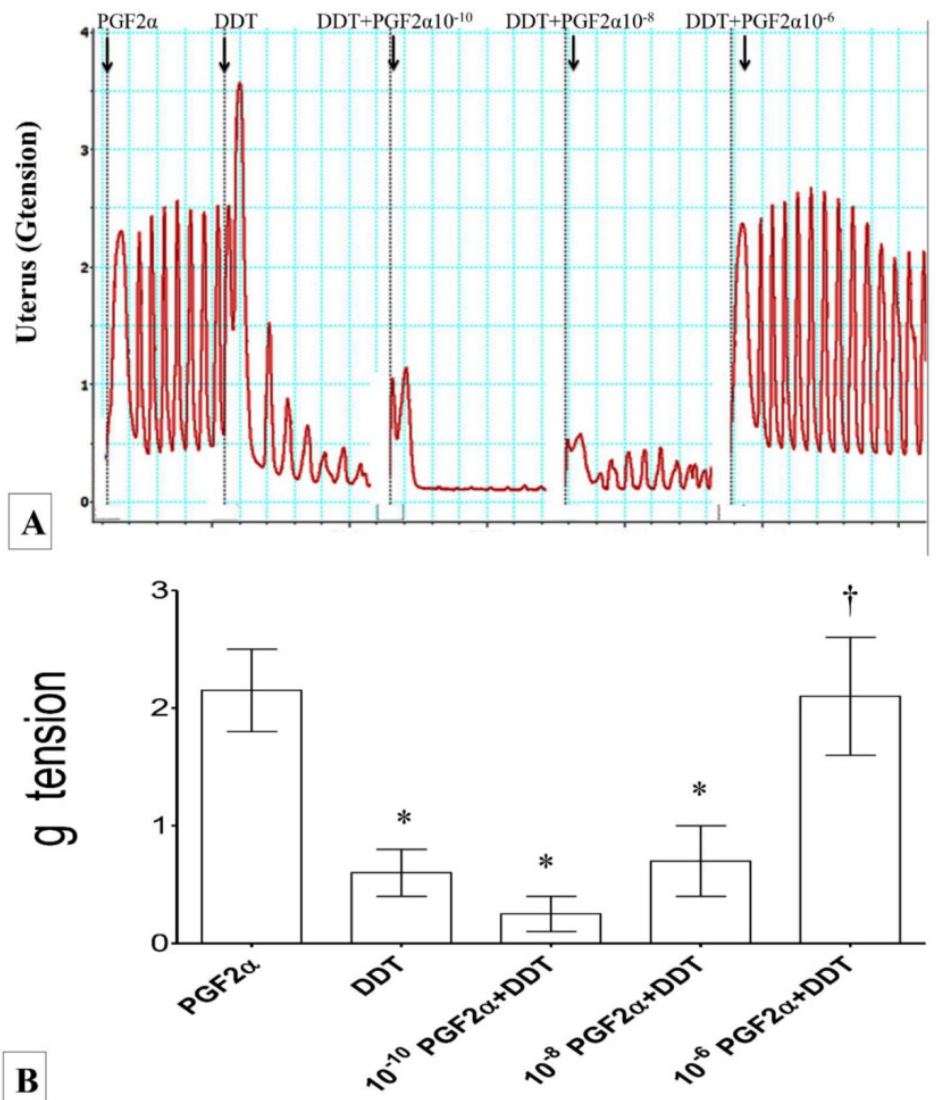

Figure 8. (A) \& (B): Combined effect of PGF2 $\alpha$ and DDT on uterine contraction. Exposure to 10-6M PGF2 $\alpha$ alone resulted in highest force of uterine contraction: Exposure to M DDT caused significant decrease in the contractile force. The force of contraction increased following administration of DDT with increasing dose of PGF2 $\alpha$. * $p<0.05$ compared to PGF2 $\alpha$. $\dagger p<0.05$ compared to DDT. $n=6$ animals per group Pros: PGF2 $\alpha$, DDT: dichrolodiphenyltrichloroethan. 

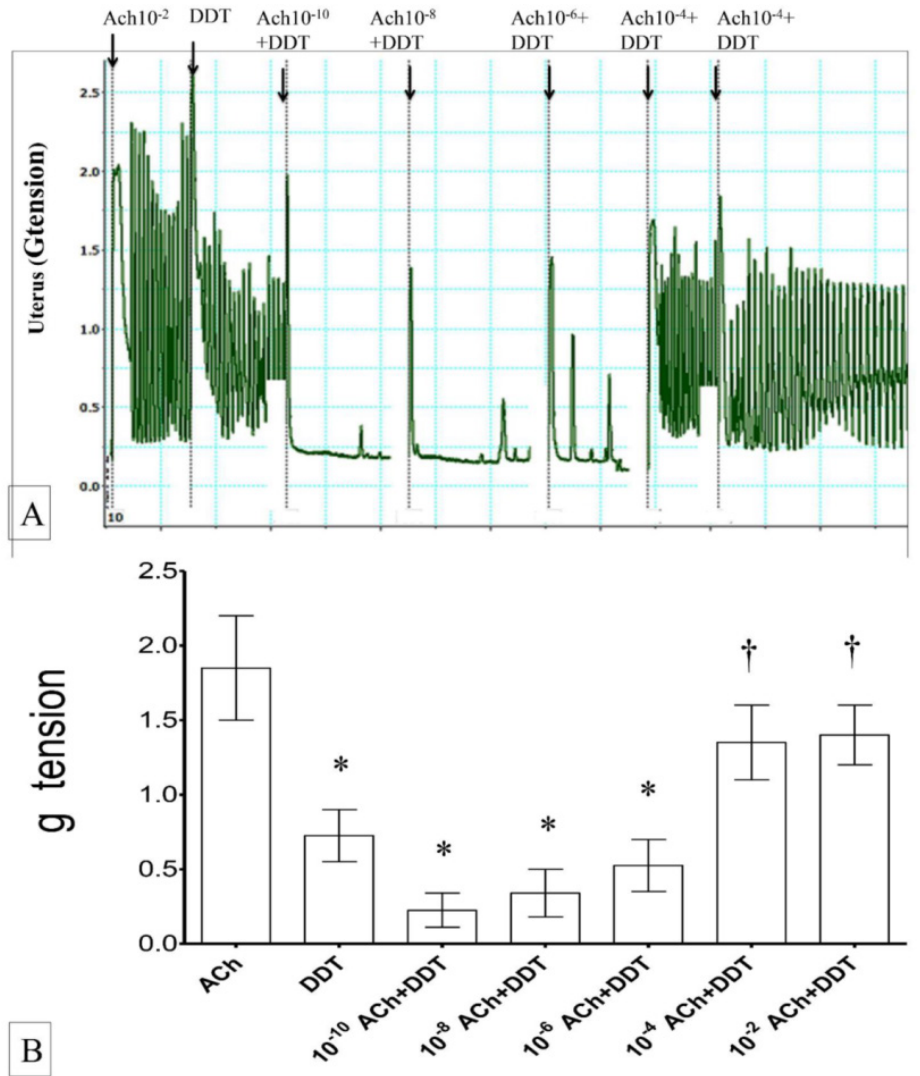

Figure 9. (A) \& (B): Combined effect of Ach and DDT on uterine contraction. Exposure to 10-2M Ach resulted in an increase in the force of uterine contraction. Exposure to DDT caused a significant increase in the contraction force which were progressively relieved upon exposure to increasing dose of Ach. * $\mathrm{p}<0.05$ as compared to ACh. $\uparrow \mathrm{p}<0.05$ as compared to DDT $n=6$ animals per group $\mathrm{ACH}$ : acetylcholine.
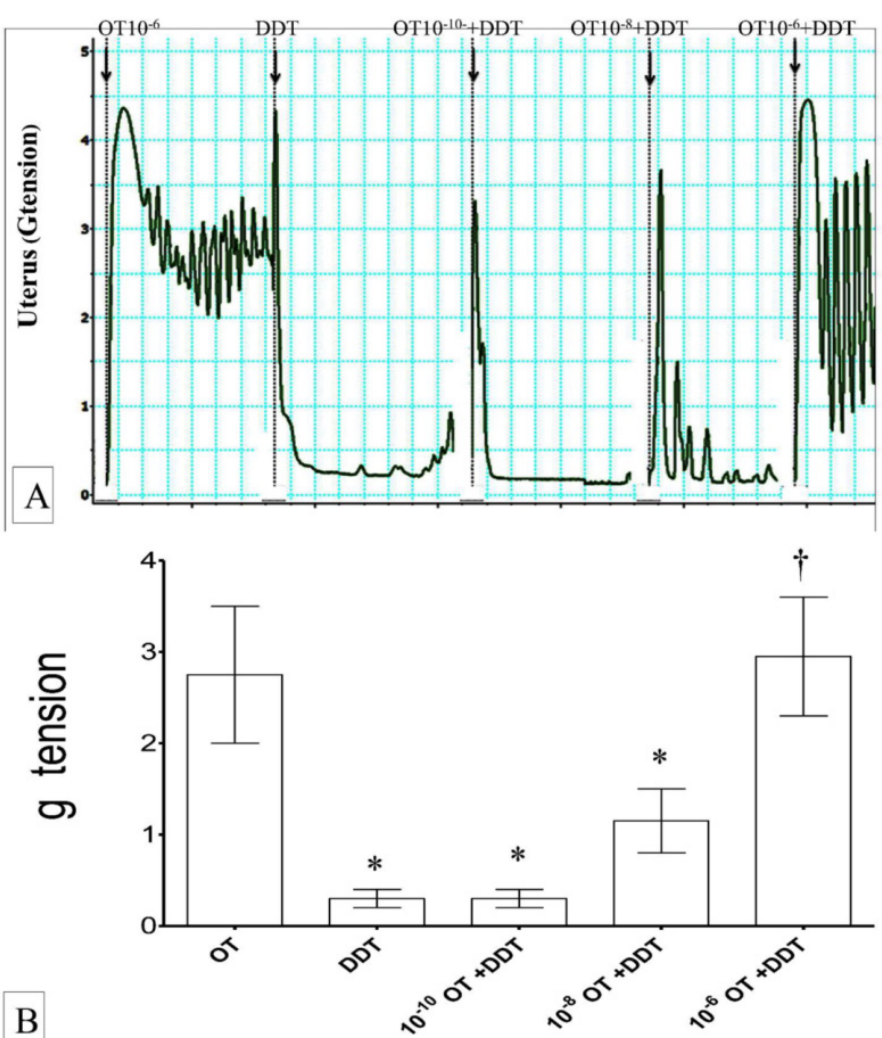

Figure 10. (A) \& (B): Combined effects of oxytocin and DDT on uterine contraction. Exposure to 10-6 M oxytocin resulted in highest uterine contractile forces. Exposed to DDT markedly reduced the force of contraction which was progressively increased following co-administration with oxytocin in a dose-dependent manner. $* p<0.05$ as compared to oxytocin. $\uparrow p<0.05$ as compared to DDT $n=6$ animals per group OT:oxytocin, DDT: dichrolodiphenyltrichloroethan. 


\section{Discussion}

This study confirmed that BPA, vinclozolin and DDT could induce abnormal contraction of the uterus. The effects of these compounds were found to involve the uterotonin pathways. Effects of uterotonins on uterine contraction have been well reported. PGF2a, Ach and oxytocin were found to stimulate uterine contraction via binding to their respective receptors i.e. prostaglandin F (FP)[20], muscarinic and oxytocic receptors respectively [21]. The ligand-receptor binding is reversible, therefore allowing the competition to occur between ligands with almost identical configuration [22]. Our preliminary findings which showed that BPA, vinclozolin and DDT decreased the uterine contractile forces indicates that these compounds work via inhibiting uterotonin actions. These could occur via binding to the uterotonin receptor. Alternatively, EDCs could also interfere with downstream pathways that was triggered upon receptor binding [23]. Uterotonin receptors are reported to be abundant in the uterus, which levels could be affected by estrogen [24]. Administration of single high dose of estrogen could help to increase the number of these receptors [24]. Following receptor binding, the effects produced were fast, therefore an immediate response could be seen. Apart from blocking the membrane receptor, endocrine disrupting chemicals (EDCs) could also interfere with $\mathrm{Ca}^{2+}$ influx and intracellular $\mathrm{Ca}^{2+}$ released, therefore decreasing uterine contractility. The involvement of membrane receptors and $\mathrm{Ca}^{2+}$ in mediating EDC effects on the uterus thus warrants further investigations.

In this study, both the force and pattern of uterine contraction were altered following BPA, vinclozolin and DDT exposures. Changes in the force were reflected by changes in $\mathrm{g}$ tension while changes in the contractile pattern were reflected by intervals between two successive spikes. Smooth muscle contraction consist of two phases: slow waves and spikes [25]. Increased number of spikes could result in increased contractile strength due to increased formation of $\mathrm{Ca}^{2+}$-calmodulin complex that activates myosin-light chain kinases [26]. EDCs might interfere with the normal contractile processes which could decrease the contractility. In addition, the contractile force could also be influence by number of action potential generated in the pacemaker cells. In a single unit smooth muscle examples uterus, the electrical potentials spread via varicosities which depolarizes the sarcolemma membrane [26]. The generation of irregular contraction indicated that the pacemaker impulse generation, synaptic transmission or sarcoplasmic membrane depolarization in uterus were impaired following EDC exposure.
We have shown that BPA could induce decreased in uterine contractile force in dose-dependent manners which were found to involve the uterotonin pathways. Additionally, changes in the pattern of contraction were also observed under BPA influence. Despite of lack of studies showing the effect of BPA on uterus, the mechanisms underlying BPA effect on the heart and nerves have been studied in detail. BPA was found to affect action potential generation in the heart and slows down electrical conduction [27] via a nitric oxide-dependent signaling pathway [28]. BPA could also modify the $\mathrm{Ca}^{2+}$ handling which could promote ventricular arrhythmia [29], increases the duration of ventricular fibrillation [30] and alters the ionic currents in the nodal cells [31]. In addition, BPA could affect cardiac muscle contraction via affecting the key regulatory proteins and ryanodine receptors [32]. A strong correlation was reported between urinary BPA level and heart rate variability in humans [33]. Meanwhile, in the nerves, inhibitory effect of $\mathrm{BPA}$ on impulse conduction has been reported which involve changes in intracellular $\mathrm{Ca}^{2+}$ [34]. BPA has been found to inhibit $\mathrm{Na}^{+}$currents via the voltage-gated sodium channel in dorsal root ganglia [35]. Several evidences indicated that BPA could produce adverse effect on the reproductive organs. BPA was found to affect the expression of calmodulin and phosphorylation of calcium/calmodulin dependent kinase in the rat testis [36]. In male rabbits, contraction and relaxation of cavernosa smooth muscles were suppressed by BPA [37]. We speculated that similar effect could occur in uterus resulting in inhibition on uterine contraction. The inhibitory effect of BPA on smooth muscle contraction were observed in in-vitro collagen gel contraction study which showed that exposure to BPA could suppress the expression of uterine contractile proteins involving oxytocin and prostaglandin related pathways [10].

To the best of our knowledge, this study was the first to demonstrate the inhibitory effect of vinclozolin on uterine smooth muscle contraction involving uterotonin pathways. Our findings revealed that vinclozolin decreased uterine smooth muscle contraction although there was an initial spike in contraction. The decrease in contractile force that follows might be due to inability of the smooth muscle to sustain phasic contraction rather than due to smooth muscle relaxation. These effects could be due to alteration in the ionic movement across the cell. Vinclozolin is a known fungicide which exerted anti-androgenic effect on the male reproductive system [38]. In neonatal male rats, exposure to vinclozolin resulted in down-regulation of expression of myosin heavy chain [39]. In females, studies have shown that vinclozolin exposure has no effect on the uterine weight and nu- 
clear sex-steroid receptor [40]. Recent studies in rats indicated that exposure to vinclozolin during gestation could induce histopathological changes in the placenta and in the maternal and fetal tissues including kidneys, heart, thymus and brain [41]. The detail mechanisms underlying vinclozolin effect on uterine smooth muscle therefore warrants further investigations.

This study has shown that DDT administration could cause decrease in uterine contractile forces and the effects involve uterotonin pathways. DDT is a common insecticide which was found to affect the uterine functions. Despite of the increased in uterine contractile force, in rats, the frequency of uterine contraction was also increased by DDT [42]. Previous studies have shown that uterine effect of DDT involve the PGE2 pathways [43]. Therefore, this finding supported our observation that PGF2a pathway was involved in mediating DDT effect. However, detail molecular mechanisms warrant further investigations.

Taken together, we concluded that exposure to BPA, vinclozolin and DDT could induce changes in the force and pattern of uterine contraction ex-vivo involving uterotonin pathways. Disturbances to uterine contractile forces and its contraction patterns could disturb the uterine functions that might lead to multiple adverse effects on reproduction. Further studies are needed to identify the specific pathways involve in mediating these compounds effects on the uterus.

\section{Acknowledgement}

This study was funded by UMRG grant (RG404/13HTM), University of Malaya, Kuala Lumpur, Malaysia.

\section{Competing Interests}

\section{Authors have nothing to disclose.}

\section{References}

1. Angioni S, Spedicato M, Rizzo A, et al. In vitro activity of human chorionic gonadotropin (hCG) on myometrium contractility. Gynecol Endocrinol. 2011; 27: $180-4$

2. Chanrachakul B, Pipkin FB, Khan RN. Contribution of coupling between human myometrial $\beta 2$-adrenoreceptor and the BKCa channel to uterine quiescence. Am J Physiol Cell Physiol. 2004; 287: C1747-C52.

3. Marshall JM. Effects of estrogen and progesterone on single uterine muscle fibers in the rat. Am J Physiol. 1959; 197: 935-42.

4. Johnson MH. Essential reproduction. John Wiley \& Sons; 2012

5. Diamanti-Kandarakis E, Bourguignon JP, Giudice LC, et al. Endocrine-disrupting chemicals: an Endocrine Society scientific statement. Endocr Rev. 2009; 30: 293-342.

6. Smith CC, Taylor HS. Xenoestrogen exposure imprints expression of genes (Hoxa10) required for normal uterine development. FASEB J. 2007; 21: 239-46.

7. Xiao S, Diao H, Smith MA, et al. Preimplantation exposure to bisphenol A (BPA) affects embryo transport, preimplantation embryo development, and uterine receptivity in mice. Reprod Toxicol. 2011; 32: 434-41.

8. Berger RG, Shaw J, decatanzaro D. Impact of acute bisphenol-A exposure upon intrauterine implantation of fertilized ova and urinary levels of progesterone and 17ß-estradiol. Reprod Toxicol. 2008; 26: 94-9.
9. Ehrlich S, Williams PL, Missmer SA, et al. Urinary bisphenol A concentrations and early reproductive health outcomes among women undergoing IVF. Hum Reprod. 2012; 27: 3583-92.

10. An BS, Ahn HJ, Kang HS, et al. Effects of estrogen and estrogenic compounds, 4-tert-octylphenol, and bisphenol A on the uterine contraction and contraction-associated proteins in rats. Mol Cell Endocrinol. 2013; 375: 27-34.

11. [Anway MD, Leathers $C$, Skinner MK. Endocrine disruptor vinclozolin induced epigenetic transgenerational adult-onset disease. Endocrinology. 2006; 147: 5515-23

12. Quignot N, Arnaud M, Robidel F, et al. Characterization of endocrine-disrupting chemicals based on hormonal balance disruption in male and female adult rats. Reprod Toxicol. 2012; 33: 339-52.

13. Laws SC, Carey SA, Kelce WR, et al. Vinclozolin does not alter progesterone receptor (PR) function in vivo despite inhibition of PR binding by its metabolites in vitro. Toxicology. 1996; 112: 173-82.

14. Lemos M, Van Gestel C, Soares A. Reproductive toxicity of the endocrine disrupters vinclozolin and bisphenol A in the terrestrial isopod Porcellio scaber (Latreille, 1804). Chemosphere. 2010; 78: 907-13.

15. Snedeker SM. Pesticides and breast cancer risk: a review of DDT, DDE, and dieldrin. Environ Health Perspect. 2001; 109: 35.

16. Venners SA, Korrick S, Xu X, et al. Preconception serum DDT and pregnancy loss: a prospective study using a biomarker of pregnancy. Am J Epidemiol. 2005; 162: 709-16.

17. Cohn BA, Cirillo PM, Wolff MS, et al. DDT and DDE exposure in mothers and time to pregnancy in daughters. Lancet. 2003;361:2205-6.

18. Bafor EE, Amogbai E.K.I, Ozolua R.I. In vitro determination of the uterine stimulatory effect of the aqueous leaf extract of Ficus exasperata. J Ethnopharmacol. 2010; 127: 502-7.

19. Oropeza MV, Monter HP, Tello TV, et al. Anatomical differences in uterine sensitivity to prostaglandin F2a and serotonin in non- pregnant rats. Eur J Pharmacol. 2002; 446: 161- 66.

20. Sugimoto Y, Yamasaki A, Segi E, et al. Failure of parturition in mice lacking the prostaglandin F receptor. Science. 1997; 277: 681-3.

21. Salleh N, Ahmad VN. In-Vitro effect of Ficus deltoidea on the contraction of isolated rat's uteri is mediated via multiple receptors binding and is dependent on extracellular calcium. BMC Complement Altern Med. 2013; 13: 359.

22. Berg J, Tymoczko JL, Stryer L. Biochemistry; $7^{\text {th }}$ edition. New York: Freeman; 2010.

23. Corbel T, Gayrard V, Puel S, et al. Bidirectional placental transfer of Bisphenol A and its main metabolite, Bisphenol A-Glucuronide, in the isolated perfused human placenta. Reprod Toxicol. 2014; 47: 51-8.

24. Dittrich R, Mueller A, Oppelt PG, et al. Differences in muscarinic-receptor agonist-, oxytocin-, and prostaglandin-induced uterine contractions. Fertil Steril. 2009; 92: 1694-700.

25. Horowitz A, Menice $C B$, Laporte $R$, et al Mechanisms of smooth muscle contraction. Physiol Rev. 1996; 76: 967-1003.

26. Hall JE. Guyton and Hall Textbook of Medical Physiology: Enhanced E-book. Elsevier Health Sciences; 2010.

27. Posnack NG, Rafael Jaimes Iii HA, Swift LM, et al. Bisphenol A exposure and cardiac electrical conduction in excised rat hearts. Environ Health Perspect. 2014; 122: 384.

28. Pant J, Ranjan P, Deshpande SB. Bisphenol A decreases atrial contractility involving NO-dependent G-cyclase signaling pathway. Journal of Applied Toxicology J Appl Toxicol. 2011; 31: 698-702.

29. Yan $S$, Chen $Y$, Dong $M$, et al. Bisphenol $A$ and $17 \beta$-estradiol promote arrhythmia in the female heart via alteration of calcium handling. PloS one. 2011; 6: e25455.

30. Yan S, Song W, Chen Y, et al. Low-dose bisphenol A and estrogen increase ventricular arrhythmias following ischemia-reperfusion in female rat hearts. Food Chem Toxicol. 2013; 56: 75-80.

31. Rubin BS. Bisphenol A: An endocrine disruptor with widespread exposure and multiple effects. J Steroid Biochem Mol Biol. 2011; 127: 27-34.

32. Gao X, Liang $Q$, Chen $Y$, et al. Molecular mechanisms underlying the rapid arrhythmogenic action of bisphenol A in female rat hearts. Endocrinology. 2013; 154: 4607-17.

33. Bae S, Kim JH, Lim YH, et al. Associations of Bisphenol A Exposure With Heart Rate Variability and Blood Pressure. Hypertension. 2012; 60: 786-93.

34. Wang W, Wang J, Wang Q, et al. Bisphenol A modulates calcium currents and intracellular calcium concentration in rat dorsal root ganglion neurons. J Membr Biol. 2013; 246: 391-7.

35. Wang $\mathrm{Q}, \mathrm{Cao} \mathrm{J}, \mathrm{Zhu} \mathrm{Q}$, et al. Inhibition of voltage-gated sodium channels by bisphenol A in mouse dorsal root ganglion neurons. Brain Res. 2011:1378: 1-8.

36. Qian W, Zhu J, Mao C, et al. Involvement of CaM-CaMKII-ERK in Bisphenol A-induced Sertoli Cell Apoptosis. Toxicology. 2014; 324: 27-34

37. Moon DG, Park HS, Cho HY, et al. Effect of Bisphenol A on the Erectile Tissues of the Rabbit. Korean J Androl. 2001; 19: 49-56.

38. Gray LE, OsTby JS, Kelce WR. Developmental effects of an environmental antiandrogen: the fungicide vinclozolin alters sex differentiation of the male rat. Toxicol Appl Pharmacol. 1994; 129: 46-52.

39. Okumu LA, Bruinton S, Braden TD, et al. Estrogen-Induced Maldevelopment of the Penis Involves Down-Regulation of Myosin Heavy Chain 11 (MYH11) Expression, a Biomarker for Smooth Muscle Cell Differentiation. Biol Reprod. 2012; $87: 1-10$. 
40. Laws SC, Carey SA, Kelce WR, et al. Vinclozolin does not alter progesterone receptor (PR) function in vivo despite inhibition of PR binding by its metabolites in vitro. Toxicology. 1996; 112: 173-82.

41. Arzu E, Süloğlu AK, Selmanoğlu G. Vinclozolin Exposure throughout Pregnancy and Its Developmental Toxicity. Toxicol Rese. 2014; 3: 375-83.

42. Juberg DR, Webb RC, Loch-Caruso R. Characterization of $\mathrm{o}, \mathrm{p}$-DDT-stimulated contraction frequency in rat uterus in vitro. Fundam Appl Toxicol. 1991; 17: 543-9.

43. Juberg DR, Loch-Caruso R. Investigation of the role of estrogenic action and prostaglandin E2 in DDT-stimulated rat uterine contractions ex vivo. Toxicology. 1992; 74: 161-72. 